TEXTSORTENBASIERTE SPRACHARBEIT

Textsortenbasierte Spracharbeit: ein Zugang zur Sprach- und

Kulturvermittlung

\author{
Renate Freudenberg-Findeisen
}

Universität Trier

\begin{abstract}
Rezeptives und produktives Textsortenwissen spielt für Lernende einer Fremdsprache eine wesentliche Rolle, denn wie die Befunde der Kognitionswissenschaft und Textlinguistik zeigen, haben sich Textsorten in einer Sprach- und Kulturgemeinschaft historisch entwickelt, um bestimmte kommunikative Aufgaben in der sozial-kulturellen Handlungspraxis zu bewältigen. Als komplexe mentale Konzepte geben Textsorten und das Wissen über sie den kommunizierenden Orientierungshilfen für das Textverstehen und die Textproduktion. Eine differenzierte Textsortenkompetenz ist damit Teil einer umfassenden Sprach- und Kulturkompetenz, die es im Fremdsprachenunterricht zu vermitteln gilt und die sich u.a. daran zeigt, wie gut Fremdsprachenlernende unterschiedliche kommunikative Situationen sprachlich adäquat bewältigen, wie gut sie unterschiedliche Texte (Zeitungsbericht, Werbeanzeige, Porträt, Ratgeber) lesen und verstehen können und wie gut es ihnen gelingt, Texte adressatenbezogen und der Situation und Intention entsprechend $\mathrm{zu}$ schreiben.

Im Fremdsprachenunterricht Deutsch und in DaF-Lehrwerken aber scheint die Arbeit an Textsorten noch immer unterschätzt zu werden. So sind zwar in vielen aktuellen DaF-Lehrwerken Textsorten ausgewiesen, was durchaus die Bemühungen der Didaktik um eine Neubewertung der Texte und Textsorten zeigt, an einer textsortenbasierten Spracharbeit aber mangelt es nach wie vor. Deshalb will der Beitrag ausgehend vom Potential, das Textsorten für eine Sprach- und Kulturdidaktik bieten, bisherigen Bemühungen um eine Textsortendidaktik DaF zusammentragen und auf dieser Grundlage aktuelle Lehrwerkauszüge beispielhaft einer kritischen Analyse unterziehen.
\end{abstract}


Textsortenbasierte Spracharbeit: ein Zugang zur Sprach- und Kulturvermittlung

Der vorliegende Beitrag beschäftigt sich mit Texten resp. Textsorten. Dabei wird zunächst der Zusammenhang zwischen Text, Textsorte und Text(sorten)kompetenz herausgestellt, um dem didaktischen Potenzial einer textsortenbasierten Spracharbeit nachzugehen. An einigen ausgewählten Zugängen, wie Prototypikalität und Musterhaftigkeit, sprachlichen Varietäten und Adressatenspezifik wie auch Kulturalität soll beispielhaft herausgearbeitet werden, warum die Kategorie der Textsorte als eine Art „didaktischer Hebel“ - wie es Beese und Roll (2015: 51) formulieren dienen kann. Dieses Potenzial gilt es für Unterricht und DaF-Lehrwerke nutzbar zu machen, ist doch der Text als kommunikative Größe sowohl Ausgangspunkt als auch Ziel des Fremdsprachenunterrichts, bildet damit gleichsam die zentrale sprachlich-kommunikative wie auch didaktische Einheit (vgl. u.a. Bausch et. al 2007, Hallet 2010, Thonhauser 2010, Neuland/Peschel 2013: 28f). Textsorten sind in den aktuellen DaFLehrwerken angekommen und sind oft auch in den inhaltlichen Modulen unter Themen und Texten eigens ausgewiesen ${ }^{1}$, was durchaus die Bemühungen der Didaktik um „eine Neubewertung der Texte und Textsorten" (Thonhauser 2010: 1033) zeigt. Nach wie vor aber mangelt es an einer textfundierten und textsortenbasierten Spracharbeit, werden „textsortenlinguistische Fragestellungen oder Perspektiven im Fremdsprachenunterricht [...] immer noch viel $\mathrm{zu}$ wenig berücksichtigt" (Fandrych/Thurmair 2011a: 351) ${ }^{2}$. Ausgehend von Beispielanalysen und einem Blick in aktuelle Lehrwerke werden im Beitrag Anforderungen und Wünsche an eine textsortenfundierte Spracharbeit formuliert.

\section{Texte, Textsorten und Text(sorten)kompetenz}

In der aktuellen textdidaktischen Debatte besteht Konsens darüber, dass Texte das zentrale Medium der Wissens-, Sprach- und Kulturvermittlung sind, die Ausbildung textueller Kompetenzen demzufolge als „Kern fremdsprachlichen Lernens“ (Hallet 2010: 173) betrachtet wird und, wie Fandrych und Thurmair, schreiben:

\footnotetext{
${ }^{1}$ Vgl. z.B. die aktuellen DaF-Lehrwerke „DaF kompakt“, „Menschen“, „Studio d“ oder auch „Aspekte“.

${ }^{2}$ Vgl. auch Stein 2004 und 2016, Venohr 2007 und Freudenberg- 


\section{TEXTSORTENBASIERTE SPRACHARBEIT}

„eine umfassende Text- bzw. Textsortenkompetenz ist eine, wenn nicht die wichtigste Kompetenz in der Sprachentwicklung, darauf haben in letzter Zeit verstärkt die Didaktik (insbesondere auch im Bereich Deutsch als Zweitsprache) und die Spracherwerbsforschung aufmerksam gemacht." (Fandrych/Thurmair 2011a: 351; vgl. dazu auch die Beiträge im Themenheft „Textkompetenz" der Zeitschrift Fremdsprache Deutsch 2008, bes. PortmannTselikas/Schmölzer-Eibinger)

Und mit dem Begriff „Textkompetenz“ wird auf Wissen und Fähigkeiten referiert: auf Wissen um Texte, auf die Fähigkeit, vorliegende Texte verstehend lesen und eigene Texte textsortenadäquat schreiben zu können, auf die Fähigkeit also, sprachlich-kommunikative Aufgaben angemessen (situations- und adressatengerecht) lösen zu können (vgl. PortmannTselikas/Schmölzer-Eibinger 2008: 5f). Textkompetenz ist damit immer auch Textsortenkompetenz.

Um die Beziehungen zwischen Einzeltext, Textsorte und sich daraus ergebenden Wissensbeständen für Rezeption und Produktion anschaulich zu machen, soll mit einem bunten Mix an Texten begonnen werden. Obwohl nur Textausschnitte auf der Folie präsentiert werden, kann man sie mühelos einzelnen Textsorten zuordnen. 


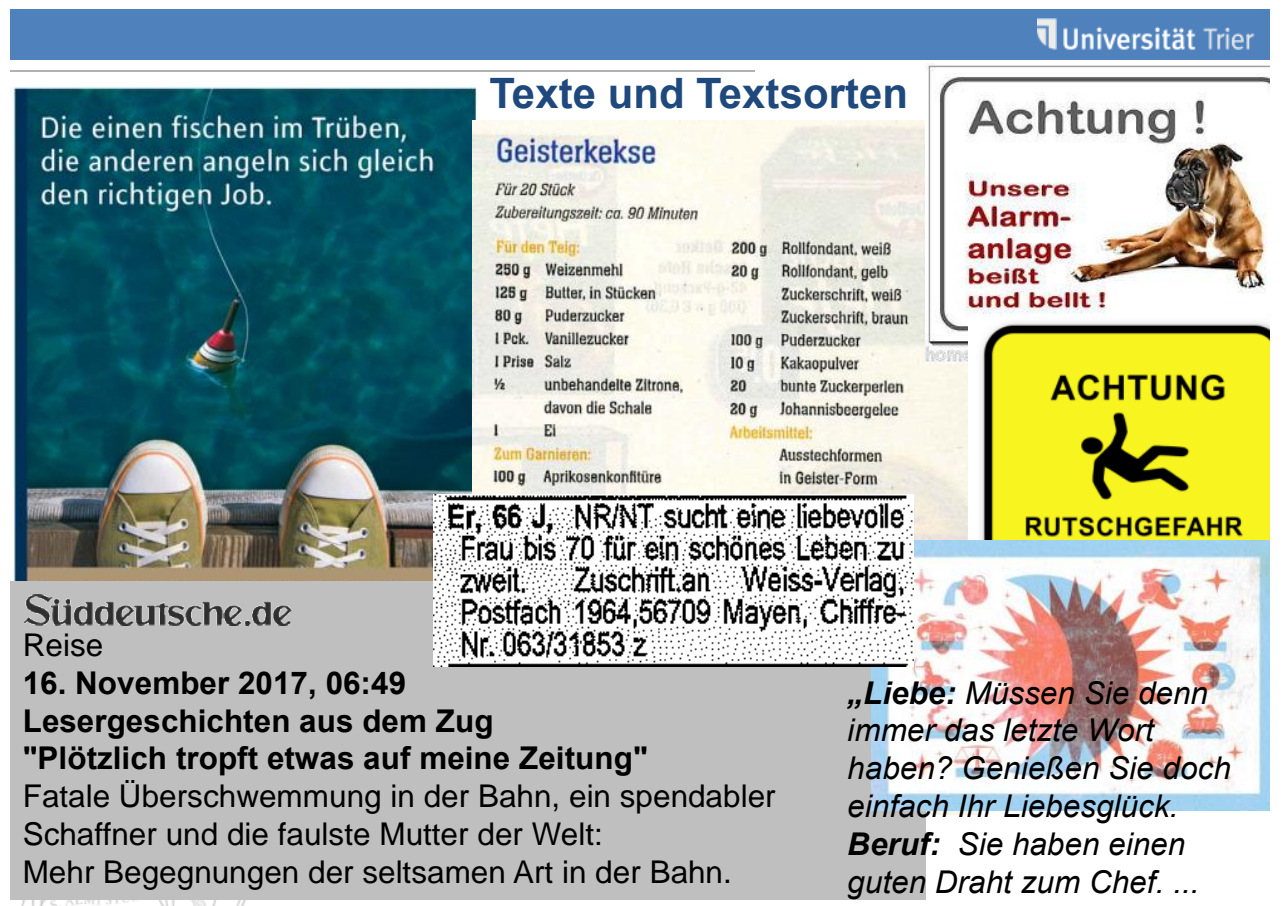

So erkennt man eine Werbeanzeige, ein Backrezept, Warnschilder, eine Kontaktanzeige, eine Lesergeschichte aus der Zeitung und einen Teil eines Horoskops. Es gibt offensichtlich Merkmale, die es dem Rezipienten ermöglichen, die einzelnen Textexemplare einer Textsorte zuzuweisen. $\mathrm{Zu}$ diesen Merkmalen gehören Besonderheiten im Layout, wie Farben, die typographische und bildliche Gestaltung, Hinweise auf die Textumgebung und damit auf die kommunikative Situation, insbesondere auf den Kanal, das Medium und die Kommunikationspartner. Aber auch Thema, Textstruktur und Textaufbau sowie spezifische Lexeme oder syntaktische Verknüpfungen gehören dazu. So lassen die Tierkreiszeichen in Verbindung mit dem typischen Textaufbau (bestimmte Kategorien wie Liebe, Beruf, Gesundheit) beispielsweise auf ein Horoskop schließen. „Süddeutsche.de“ verweist auf die bekannte überregionale Süddeutsche Zeitung, insbesondere auf ihre Onlineausgabe, und die Überschriften auf eine bestimmte Rubrik - hier Reise - und einen dazu gehörigen Artikel. Das Backrezept wie auch den Zeitungsartikel erkennt man an deren typischer Struktur (Zutatenliste; Rubrik, Überschrift, Unterüberschrift), die Warnschilder zum Teil an ihrer Farbe wie auch an „warnenden“ Lexemen, die Kontaktanzeige an der typischen Syntax und die Werbung an der BildText-Gestaltung und dem Werbespruch. 


\section{TEXTSORTENBASIERTE SPRACHARBEIT}

Texte präsentieren, wie Stein es formuliert, „immer bestimmte Textsorten, die sich aufgrund gemeinsamer Eigenschaften ergeben und sich im konkreten Textexemplar mehr oder weniger deutlich widerspiegeln können“ (Stein 2004: 179). Der Begriff bzw. die Kategorie Textsorte wird in der Linguistik allgemein, kommunikativ-funktional wie auch prototypisch gefasst. So sind Textsorten allgemein,,eine finite Menge von Textexemplaren mit spezifischen Gemeinsamkeiten“ (Heinemann 2000: 519), die man auch als „Basisklassen von Texten“ (Heinemann/Heinemann 2002: 143) bestimmen kann. Textsorten haben sich in einer Sprach- und Kulturgemeinschaft historisch entwickelt, um bestimmte wiederkehrende kommunikative Aufgaben in der sozialen Handlungspraxis zu bewältigen. Damit gehören sie zum Alltagswissen, sind zweckbestimmt und haben eine kulturelle Dimension. Sie besitzen textuelle bzw. kulturelle Besonderheiten der jeweiligen Sprachgemeinschaft. Das kommt zuvörderst in ihrer Prototypik zum Ausdruck: So teilen die Exemplare einer Textsorte prototypische textinterne und textexterne Merkmale, d.h. sie haben Gemeinsamkeiten im Textaufbau, in der Textstruktur sowie in der Verwendung bestimmter grammatischer, lexikalischer und idiomatischer Mittel wie hinsichtlich ihrer kommunikativen Situation und Textfunktion. (vgl. stellvertretend Brinker2010 und Heinemann 2000, Heinemann/Heinemann 2002 und Stein 2016)

Der funktional-kommunikativ und prototypisch gefassteBegriff Textsorte verweist damit gleichsam auch auf zwei unterschiedliche Kompetenzen der Sprachteilhaber: zum einen auf die Kompetenz, Texte aufgrund gemeinsamer Eigenschaften einer bestimmten Textsorte zuordnen $\mathrm{zu}$ können und zum anderen darauf, kompetent und angemessen mittels Texten handeln zu können (vgl. stellvertretend Heinemann/Heinemann 2002: 129ff). Wie an den eingangs aufgeführten Beispielen gezeigt, steuert Wissen über invariante Eigenschaften, musterhafte Textstrukturen und Formulierungen das Erkennen von Texten resp. Textsorten und führt $\mathrm{zu}$ einer entsprechenden Erwartungshaltung beim Verstehen. In Hinblick auf die Produktion ist Texthandlungswissen, wie Stein formuliert, ,als das Wissen $\mathrm{zu}$ verstehen, das die zur Lösung kommunikativer Aufgaben erforderlichen Orientierungsmuster für die Produktion [...] von Texten beinhaltet" (Stein 2016: 29).

Für die Sprachdidaktik DaF ergibt sich daraus, Texte als Vertreter einer Textsorte wahrzunehmen und in ihrem spezifischen Potenzial für Lernende entsprechend aufzubereiten. „Im Blick auf Textsorten“, so Stein, „können 
also sprachliche, strukturelle, funktional-pragmatische, kognitive und soziale bzw. soziokulturelle Aspektevon Texten im Zusammenhang betrachtet werden" (ebd.: 30). Entsprechend konzentrieren sich die textsortendidaktischen Bemühungen ${ }^{3}$ in jüngster Zeit darauf, diese Zusammenhänge für den Aufbau einer Text(sorten)kompetenz lehr- und lernbar zu machen. Einig ist man sich in der Sprachdidaktik DaF darüber, dass Textsortenkompetenz eine Summe von Wissen und Fähigkeiten bildet, d.h. dass Textsortenkompetenz eine Rezeptions- und Produktionskompetenz umfasst und auch Wissen über die in den unterschiedlichen Textsorten verwendeten spezifischen grammatischen und lexikalischen Mittel wie auch kulturspezifische Aspekte einschließt.

Sprachdidaktisch besonders innovativ erscheinendabei folgende Zugänge:

- Zusammenhänge zwischen funktionalen und strukturellen Aspekten: Prototypik und Musterhaftigkeit

Die Arbeit mit Textsorten erlaubt den Lernenden Einsicht darin, wie der mit einer Text-sorte verbundene kommunikative Zweck realisiert werden kann. Auf diese Weise bleiben sprachliche resp. textuelle Mittel nicht abstrakt und isoliert, sondern werden als Werkzeuge wahrgenommenund es wird möglich, die Verbindung von Form, Bedeutung, Funktion und sprachlichem Handeln herzustellen (vgl. Fandrych/Thurmair 2011a, FreudenbergFindeisen 2014). Damit entsteht ein Wissen über invariante Eigenschaften, musterhafte Textstrukturen wie auch typische Formulierungen. Ein Ziel der Sprachvermittlung sollte es sein, „Muster für möglichst viele Textsorten in den Köpfen der Lernenden zu installieren“ (Feld-Knapp 2005: 120).

- Textsorten als Möglichkeit, sprachliche Varietäten und aktuelle Sprachwandelprozesse in den Unterricht zu bringen

Das Deutsche ist sowohl eine lebendige, sich ständig verändernde Sprache als auch durch eine Vielfalt an sprachlichen Ausdrucksformen gekennzeichnet, die sich „begrifflich als ein Neben- und Miteinander von unterschiedlichen Varietäten und Stilen begreifen“" (Spiekermann 2010: 344) lassen. Diesen Umstand muss ein auf die Ausbildung von kommunikativ essentiellen Kompetenzen ausgerichteter DaF-Unterricht

\footnotetext{
${ }^{3}$ Vgl. z.B. die Sammelbände von Bachmann/Feilke 2014, Benholz/Frank/Gürsoy 2015 und Freudenberg-Findeisen 2016. 


\section{TEXTSORTENBASIERTE SPRACHARBEIT}

berücksichtigen. Und deshalb scheint es auch kein Zufall zu sein, dass gerade in den letzten Jahren immer wieder auch Auslandsgermanisten nachdrücklich darauf hingewiesen haben, dass die Standardsprache nicht einziger Gegenstand und Ziel des DaF-Unterrichts sein kann ${ }^{4}$. Es muss vielmehr auch den Varietäten, die in der alltagssprachlichen Wirklichkeit Verwendung finden, im fremdsprachlichen Deutschunterricht Beachtung geschenkt werden.

Gerade die Arbeit mit Texten resp. Textsorten kann den Blick für sprachliche Variation öffnen, denn in Texten - wie auch an den eingangs vorgestellten Beispielen erkennbar - begegnen den Lernenden Stile, die man als eher alltagssprachlich, mediensprachlich, fachsprachlich, wissenschaftssprachlich, gesprochen, geschrieben, formell, informell oder auch als regional charakterisieren kann. Durch Textsorten kann man auf diese verschiedenen Sprachgebrauchsgewohnheiten aufmerksam machen und ein entsprechendes Gespür und Bewusstsein für unterschiedliche Varietäten und Normen schaffen.

Dass authentisches bzw. semiauthentisches Material, also Material, das die relevanten textsortenspezifischen Merkmale enthält, für eine solche funktional ausgerichtete Sprachvermittlung am ehesten eine Gewähr bietet, liegt auf der Hand und wird in der Sprachdidaktik ebenfalls immer wieder hervorgehoben, bietet dieses Material doch einen reichhaltigen, echten und glaubwürdigen Kontext, in dem Sprache situationsbezogen verwendet wird.

- Schnittstellenfunktion: Verknüpfung mit einer funktional ausgerichteten Arbeit an grammatischen Strukturen und am Wortschatz

Aktuelle Ansätze der Grammatik- wie auch Wortschatzdidaktik ${ }^{5}$ fordern mit Bezug auf gedächtnis- und lernpsychologische Erkenntnisse, grammatische Strukturen wie auch den Wortschatz nicht kontextlos bzw. situationslosgelöst und verwendungsunabhängig $\mathrm{zu}$ vermitteln, sondern beide Kenntnisbereiche im Rahmen ihrer kontextuellen Einbettung vorzustellen. Der Textsortenansatz erlaubt es, sowohl grammatische Strukturen als auch den Wortschatz in ihrem ,natürlichen Umfeld“ einzuführen. So erschließen sich erst im Text Bedeutung und

\footnotetext{
${ }^{4}$ Vgl. dazu bes. die Beiträge von Neuland, Durell und Berend/Knipf-Komlósi in Neuland 2006.

${ }^{5}$ Vgl. u.a. Kühn 2013, Fandych 2010, Decke-Cornill/Küster 2010.
} 
Verwendung eines Wortes, können durch die kontextuelle und situative Einbettung Beziehungen zwischen den Wörtern - orientiert an verschiedenen Vernetzungsarten (textbasiertes Sachnetz, Kollokationsnetz, paradigmatisches Netz usw.) - sichtbar gemacht werden wie auch

„bestimmte Wörter, Wendungen und Konstruktionen, die für bestimmte Textsorten einer bestimmten Domäne in einer bestimmten Kultur einschlägig sind, weil sie für zentrale Textfunktionen stehen" (Steinhoff/Sorrentino 2010: 192f),

herausgearbeitet werden. ${ }^{6}$ Hinsichtlich der Grammatik ist der „textsortenbezogene Grammatikansatz“, wie er von Fandrych und Thurmair (vgl. bes. Fandrych/Thurmair 2011a und b) entwickelt wurde, hervorzuheben, der grammatische Strukturen in Abhängigkeit zu ihrer Textfunktion und ihrem Zweck herausarbeitet und erklärt. Denn „Textsorten [...] schaffen einen kommunikativen Rahmen, innerhalb dessen grammatische Strukturen ihre je spezifische Wirkung entfalten“ (Fandrych/Thurmair 2011a: 345, vgl. auch FreudenbergFindeisen 2013).

- Kulturhaftigkeit und Kulturalität von Textsorten

Als über einen langen Zeitraum in einer Sprachgemeinschaft entstanden, haben Textsorten eine kulturelle Dimension, besitzen also textuelle Merkmale bzw. kulturelle Besonderheiten der jeweiligen Sprachgemeinschaft. Für den fremdsprachlichen Deutschunterricht erwächst daraus ein überaus interessantes und anspruchsvolles Anforderungsprofil, denn es gilt, das unterschiedliche Aussehen von Textsorten zwischen den Kulturen (äquivalente, partiell äquivalente wie auch äquivalentlose Formen) den Lernenden bewusst zu machen wie auch kulturelles Wissen über die Zielkultur zu vermitteln, letztlich interkulturelles Verstehen zu fördern (vgl. u.a. Feld-Knapp 2005, Hufeisen 2008).

Ein Beispiel für eine äquivalentlose Form ist auch auf der eingangs abgebildeten Folie zu finden, nämlich die Kontaktanzeige. Während sie in vielen Kulturen, so der europäischen, nordamerikanischen und auch asiatischen, eine typische Textsorte darstellt ${ }^{7}$, ist sie in der arabischen Kultur wohl kaum zu finden. Und wenn man sie findet - so sagten mir

${ }^{6}$ Vgl. dazu auch Krames 2016.

${ }^{7}$ Vgl. dazu Zhang Wei 2011 


\section{TEXTSORTENBASIERTE SPRACHARBEIT}

jordanische Studierende in einem meiner Seminare, dann seien sie ironisch oder satirisch zu interpretieren.

- Textsorten und ihre Vernetzung

Texte bzw. Textsorten stehen niemals isoliert, sondern sind in umfassendere kommunikative Strukturen eingebunden und damit Teil einer funktionalen wie thematischen Vernetzung, die in der Textlinguistik als Textsortennetz oder Textsortenfeld bezeichnet wird (vgl. Adamzik 2011: 369ff). Funktionale wie thematische Beziehungen bestehen z.B. zwischen Abstract und Artikel, Zeitungsbericht und Leserbrief, Stellenanzeige und Bewerbung oder zwischen verschiedenen ,parallelen“ Textsorten, die eine gemeinsame Intention verbindet. So können beispielsweise Informationen über eine Stadt in Form eines Lexikoneintrages, einer Webseite des Verkehrsamts, eines Artikels in einem Reisemagazin, einer Werbeanzeige oder auch in Form eines Reiseblogs vermittelt werden. Für die Sprachdidaktik eröffnet dieser Zugriff das Herausarbeiten bestimmter innerer Zusammenhänge und auch Abfolgen (welcher Text geht einem Text voraus, welcher Text ist erwartbar) wie das Sichtbarmachen von situations- und insbesondere adressatenspezifischen textuellen Merkmalen (vgl. Fandrych/Thurmair 2011a: 341ff).

Einig ist man sich in der Sprachdidaktik darüber, dass die für Lehr- und Lernzwecke ausgewählten Texte als „Prototypen für verschiedene Textsorten fungieren“ (Feld-Knapp 2005: 115, vgl. auch Stein 2004: 201ff) und damit dem Kriterium der Repräsentativität und Musterhaftigkeit ein besonderes Gewicht für die Textauswahl beigemessen wird neben folgenden, nicht minder wichtigen Kriterien: Ergiebigkeit, Authentizität, Lernerbezug mit Verweisen auf Vorwissen und Interessen, Lernziele und Unterrichtskontext (vgl. ebd. und Portmann-Tselikas 2000). Natürlich gilt es bei der Arbeit mit Textsorten abzuwägen, welche Texte resp. Sorten für welche Lerngruppen, Niveaustufen und Lernziele zentral sind. 


\section{Textsorte als didaktischer Hebel}

Die sich über Textsorten ergebenden vielfältigen Zugänge können zusammenfassend so dargestellt werden:

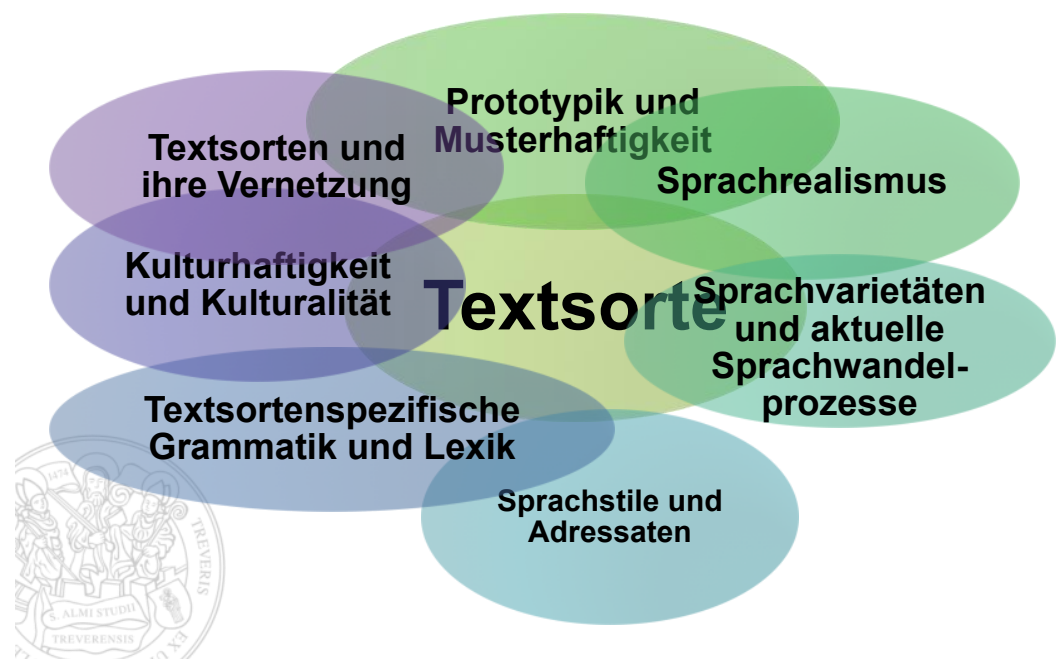

Hinsichtlich ihres Potenzials für eine textsortenbasierte Spracharbeit sollen nun die Textsorten „Wetterbericht", „Webseite zu einer Stadt" und „Reiseblog ${ }^{\star 8}$ näher analysiert und kommentiert werden. Diese Textsorten wurden gewählt, weil sie zu wesentlichen Kommunikationsbereichen des Alltags und der Kultur gehören, also von allgemeinem Interesse sind. Sie sind vor allem rezeptive Textsorten und aus fremdsprachlicher Sicht mit nicht wenigen Anforderungen an die Verstehenskompetenz verknüpft. Die Themen sind aber auch für produktive Zwecke wesentlich, denn das Wetter wie auch Reisen bzw. das Kennen, Nicht-Kennen oder Noch-Nicht-Kennen von Ländern, Städten, Regionen etc. nehmen in vielen kommunikativen Situationen, wie der ersten Begegnung und Aufnahme von Kontakten und/oder der Kontakterhaltung, einen wichtigen Platz ein.

\footnotetext{
${ }^{8}$ Webseiten und Blogs werden in der Textsortenlinguistik eher als Kommunikationsformen betrachtet, vgl. dazu Bittner 2003 und Schlobinski/Siever 2005; einzelne Seiten wie auch thematische Blogeinträge lassen sich aber textsortenlinguistisch 
TEXTSORTENBASIERTE SPRACHARBEIT

\section{1 „Zum Teil kräftige Regengüsse mit Blitz und Donner" "9. Prototypikalität und Musterhaftigkeit von Wetterberichten}

Wie bisherige linguistische Arbeiten zu Wetterberichten ausführen ${ }^{10}$, kommen sie geschrieben (Zeitungen, Internet) oder gesprochen (Hörfunk, Fernsehen) vor, werden von visuellen Elementen (z.B. Wetterkarten, Symbolen, Zahlen) unterstützt, sind dominant wissensbezogene, informative Texte und werden von Fachleuten, Meteorologen und Klimaforschern, in Zusammenarbeit mit Journalisten erstellt, und wenden sich an alle Rezipienten. Je nach Bedürfnis der Rezipienten gibt es neben allgemeinen auch spezialisierte Wetterberichte, wie den Seewetterbericht oder das Wander- bzw. Bergwetter.Wichtig sind natürlich auch - wie gerade im Moment - Informationen zu ungewöhnlichen Wetterlagen und ihren Folgen, wie Dauerregen, Stürme, Hochwasser und Überschwemmungen, Unwetter also.

$\mathrm{Zu}$ ergänzen ist sicherlich auch noch die Wetter-App, die immer wichtiger $\mathrm{zu}$ werden scheint, da sie einen permanenten Zugriff auf Wetterentwicklungen rund um den Globus erlaubt und aufgrund verständlicher Visualisierungen für den Rezipienten leichter zugänglich ist.

Die folgende Analyse rückt Wetterberichte aus einer überregionalen wie auch regionalen Zeitung in den Mittelpunkt der Betrachtung und wird neben Unterschieden vor allem die Ähnlichkeiten aufzeigen, um Prototypisches hinsichtlich des Textaufbaus und seiner inneren Gliederung wie auch grammatischer Strukturen und lexikalischer Mittel herauszustellen. Zunächst exemplarisch zwei vollständige Beispiele ${ }^{11}$ :

\footnotetext{
${ }^{9}$ Süddeutsche Zeitung. 2/3. Juni 2018.

${ }^{10}$ Vgl. v.a. Spillner 1983, Brinker 2010 und Fandrych/Thurmair 2011a.

${ }^{11}$ Die Schreibung in den Wetterberichten entspricht den Schreibweisen der Zeitungen.
} 


\begin{tabular}{|l|}
\hline Trierischer Volksfreund \\
Donnerstag, 16. November 2017 \\
\hline Wetterumschwung am \\
Wochenende \\
Vor allem im Süden Flockenwirbel \\
Das Wochenende bringt einen \\
Wetterumschwung mit Regen, im \\
Süden und in den Mittelgebirgen \\
auch Schnee bis in tiefe Lagen. Auch \\
die neue Woche beginnt unterkühlt \\
und wechselhaft.
\end{tabular}

\section{Das Wetter von heute in Trier}

In Trier bleibtder Himmel bis zum Nachmittag grau und es regnet bei Temperaturen von 10 bis $\mathrm{zu} 15^{\circ} \mathrm{C}$. Abends ist es in Trier teils wolkig und teils heiter bei Temperaturen von 10 bis $12^{\circ} \mathrm{C}$. In der Nacht bilden sich leichte Wolken bei Tiefstwerten von $9^{\circ} \mathrm{C}$. Der Wind weht aus südlicher Richtung mit Geschwindigkeiten von 13 bis $19 \mathrm{~km} / \mathrm{h}$. Mit Böen zwischen 31 und $44 \mathrm{~km} / \mathrm{h}$ ist $\mathrm{zu}$ rechnen.

\section{Süddeutsche Zeitung}

Mittwoch, 23. Mai 2018

\section{Heiter bis wolkig, in der Mitte} und im Süden Gewitter

\section{Wetterlage}

Ein Hoch über Skandinavien lenkt warme, aber teilweise feuchte Luft aus Osteuropa nach Mitteleuropa. So steigen die Temperaturen dort örtlich bis $25 \mathrm{Grad}$, vor allem im südlichen Mitteleuropa gibt es aber einige Schauer oder Gewitter. Wechselhaftes Wetter mit Schauern und Gewitter findet man auch in den Alpenländern, in Frankreich und auf dem Balkan. Dagegen herrscht in Südostspanien sonniges und warmes Wetter.

\section{Aussichten}

Im Norden und Nordosten setzt sich das freundliche Wetter mit viel Sonnenschein fort. Dort bleibt es trocken. In der Mitte und im Süden ist es wechselhafter, und es gibt im Laufe des Tages zum Teil kräftige Regengüsse und Gewitter, örtlich sind Hagel und Sturmböen dabei. Die Höchstwerte liegen zwischen 19 und 26 Grad. Der Wind weht schwach bis mäßig, an der Küste und in den Mittelgebirgen auch frisch aus Nordost bis Ost. 


\section{TEXTSORTENBASIERTE SPRACHARBEIT}

Wie man erkennen kann, ähneln sich beide Wetterberichte schon im Textaufbau: So sind beide Berichte deutlich zweigeteilt: In einem ersten Teil erfolgt die Beschreibung der allgemeinen Wetterlage, in dem ein Überblick über das Gesamtgeschehen gegeben wie auch Wetterentwicklungen für die nahe Zukunft beschrieben werden. Je nachdem, welches Gebiet der Wetterbericht abdeckt - hier einmal ein Stadtgebiet „Trier und Umgebung“ und zum anderen den Süden Deutschlands und damit eine größere Region - kann dieser Teil ausführlicher oder auch kürzer sein. Inhaltlich bildet die Beschreibung der Wetterlage die Grundlage für den zweiten Teil, in dem dann das Wetter für den Tag bzw. die Wettervorhersage für das betreffende Gebiet gegeben wird.

In der überregionalen Zeitung werden die beiden Teile schon im Schriftbild durch die in jedem Wetterbericht wiederkehrenden Überschriften „Wetterlage“ und „Aussichten“ deutlich markiert. Eine Gesamtüberschrift, die entsprechend den Wetterverhältnissen inhaltlich variiert, in der Form aber ähnlich ist, informiert zusammenfassend über das Wettergeschehen: „Heiter bis wolkig, in der Mitte und im Süden Gewitter“, „Zum Teil kräftige Regengüsse mit Blitz und Donner" oder „Erneut einige Regengüsse und Gewitter"12. Auch in der regionalen Zeitung findet man eine Markierung beider Teile schon durch das Schriftbild, hier leitet die zusammenfassende Überschrift gleichzeitig den ersten Teil des Textes ein, der zweite Teil wird mit der stets wiederkehrenden Überschrift,,Das Wetter von heute in Trier" eingeleitet.

Neben diesem zweigeteilten Textaufbau und den Überschriften fallen weitere charakteristische sprachliche Elemente ins Auge. So sind beide Texte durch interne Gliederungen bestimmt, die Fandrych/Thurmairals „lokal, temporal und sachlich-thematisch“ (Fandrych/Thurmair 2011a: 168) fassen. Im regionalen Wetterbericht für Trier dominiert in beiden Teilen neben der lokalen und sachlich-thematischen eine temporale Gliederung entsprechend der natürlichen Chronologie des Tages und weiterer Tage. Diese Chronologie wird durch temporale Lexeme und Wortgruppen wie Wochenende, neue Woche, bis zum Nachmittag, in der Nacht oder temporale Adverbien wie abends ausgedrückt. Im Wetterbericht der Süddeutschen Zeitung dagegen dominiert neben der temporalen und sachlich-thematischen Gliederung die lokale, die sprachlich durch geographische Marker (Himmelsrichtungen, Länder, Gebiete, Städte, Gebirge, Flüsse) und ihre

\footnotetext{
${ }^{12}$ Die Beispiele sind der Süddeutschen Zeitung vom 20. bis 31. Mai 2018 entnommen.
} 
entsprechenden Verbalisierungen wie lokale Lexeme und Wortgruppen Osten, Süden, Skandinavien, Osteuropa, südliches Mitteleuropa, Alpenländer, Balkan, Küste, Trier, bis in tiefe Lagen oder lokale Adverbien wie örtlich zum Ausdruck gebracht wird. Sachlich-thematisch werden in beiden Texten zunächst die Wettererscheinungen, also Regen, Schnee, Hagel, Wolken, Sonne etc., betrachtet, danach erfolgen Angaben zu den Temperaturen und den Windverhältnissen.

Bei den Verbalisierungen für Wettererscheinungen fallen feste Kollokationen wie heiter bis wolkig, teils wolkig und teils heiter, leichte Wolken, teilweise feuchte Luft, wechselhaftes/sonniges und warmes/freundliches Wetter, kräftige Regengüsse und Gewitter,die teilsfachsprachliche Bedeutung haben, wie z.B. Hagel oder Böen, besonders auf. Die Temperaturangaben werden oft in Verbindung mit den Verben steigen und liegen bzw. durch adverbiale Anschlüsse ausgedrückt: $b e i$ Temperaturen von, die Temperaturen steigen auf ..., liegen zwischen. Zum Ausdruck der Windverhältnisse wird meist das Verb wehen in Verbindung mit Richtungs- oder Angaben der Art und Weise genutzt: der Wind weht aus südlicher Richtung/weht schwach bis mäßig/auch frisch.

Mit diesen Gliederungen verbinden sich also lexikalische Charakteristika. So zeichnen sich Wetterberichte durch thematische Wortfelder aus, die als geographisch-landes- und kulturkundlich, temporal und meteorologisch gefasst werden können. Neben den festen Kollokationen kann man auch bildliche Sprache entdecken, wie metaphorische Ausdrücke, so im o.g. Beispiel Flockenwirbel.

In Hinblick auf die Prädikationen haben Fandrych/Thurmair für Wetterberichte herausgearbeitet, dass ,die Informationen vor allem nominal realisiert" (Fandrych/Thurmair 2011a: 171) werden. Im Verbbereich begegnet man neben einigen Witterungsverben, wie wehen, regnen oder schneien, besonders oft allgemeineren Existenzverben, verbunden mit nominalen Gruppen (vgl. ebd.), so wie auch in den Beispieltexten z.B. bringt einen Wetterumschwung, bleibtder Himmel grau, bilden sich leichte Wolken, gibt es Schauer oder Gewitter, herrscht warmes und sonniges Wetter. Diese Existenzverben drücken Zustände wie auch Entwicklungen für die Zukunft und Zukünftiges aus. Sie sind damit ein „textsortenspezifisches Kennzeichen“ (ebd.: 172), denn das Tempus Futur wird in Wetterberichten nicht verwendet. 


\title{
TEXTSORTENBASIERTE SPRACHARBEIT
}

Die Beispielanalysen bestätigen die Befunde von Fandrych/Thurmair, die zu dem Ergebnis kommen, dass die Textsorte „Wetterbericht“ eine erwartbare Struktur aufweist:

\begin{abstract}
„Der Text schreitet von der Beschreibung zur Vorhersage und innerhalb dieser wird intern temporal, geographisch und nach Wetterphänomenen gegliedert, wobei hier die Abfolge variieren kann. Man kann aber dennoch von einer klar umrissenen Textsorte sprechen, der Wetterbericht kann fast als formelhafter Text gelten" (ebd.: 169).
\end{abstract}

Methodisch-didaktische Schlussfolgerungen sind für die Behandlung der Textsorte „Wetterbericht" hinsichtlich des Aufbaus eines differenzierten Verstehens zu ziehen. In der Regel liest oder hört man Wetterberichte, um sich über das Wetter an einem bestimmten Ort zu einer bestimmten Zeit zu informieren, dafür sind besonders selektive Lese- bzw. Hörstrategien notwendig. Kenntnisse über den typischen Textaufbau, seine internen Gliederungen und die entsprechenden sprachlichen Mittel können für die Ausbildung dieser Lese- und Hörfertigkeit als zentrale, das Verstehen steuernde Elemente betrachtet werden, denen bei der Aufbereitung der Texte wie auch im Vermittlungsprozess Aufmerksamkeit zu schenken ist. Im letzten Teil des Beitrages wird darauf zurückgekommen. Nicht unerwähnt sollen aus didaktischer Sicht auch die in Wetterberichten gehäuft vorkommenden geographisch-landes- und kulturkundlichen Lexeme bleiben, mit denen die Topographie der deutschsprachigen Länder wie ihre Benennungspraxis ein Stück weit eingefangen werden können, wobei natürlich eingeräumt werden muss, dass Wetterberichte nicht als spannendste Textsorte gelten.

\subsection{Deutschlandjäger und Porta Nigra - Webseite zur Stadt Trier und Reiseblog Tier: Textsorten und Adressatenspezifik}

Die nächsten zwei Beispiele rücken parallele Textsorten - den Eintrag zu Trier im Reiseblog „Deutschlandjäger. Dein Deutschland-Reiseblog \# 1“ und die Webseite der Stadt Trier - ins Zentrum. Beide verbindet eine gemeinsame Intention, sie möchten Informationen über Trier vermitteln. Und wie man an den teilweise ähnlichen Rubriken in den Menüleisten erkennen kann, wollen beide Medienformate informieren (über das Reiseziel, seine Sehenswürdigkeiten und die Region), instruieren (wie sollte 
man das gewünschte Ziel erkunden), empfehlen (was ist besonders lohnend) und nicht zuletzt für das Reiseziel einnehmen.

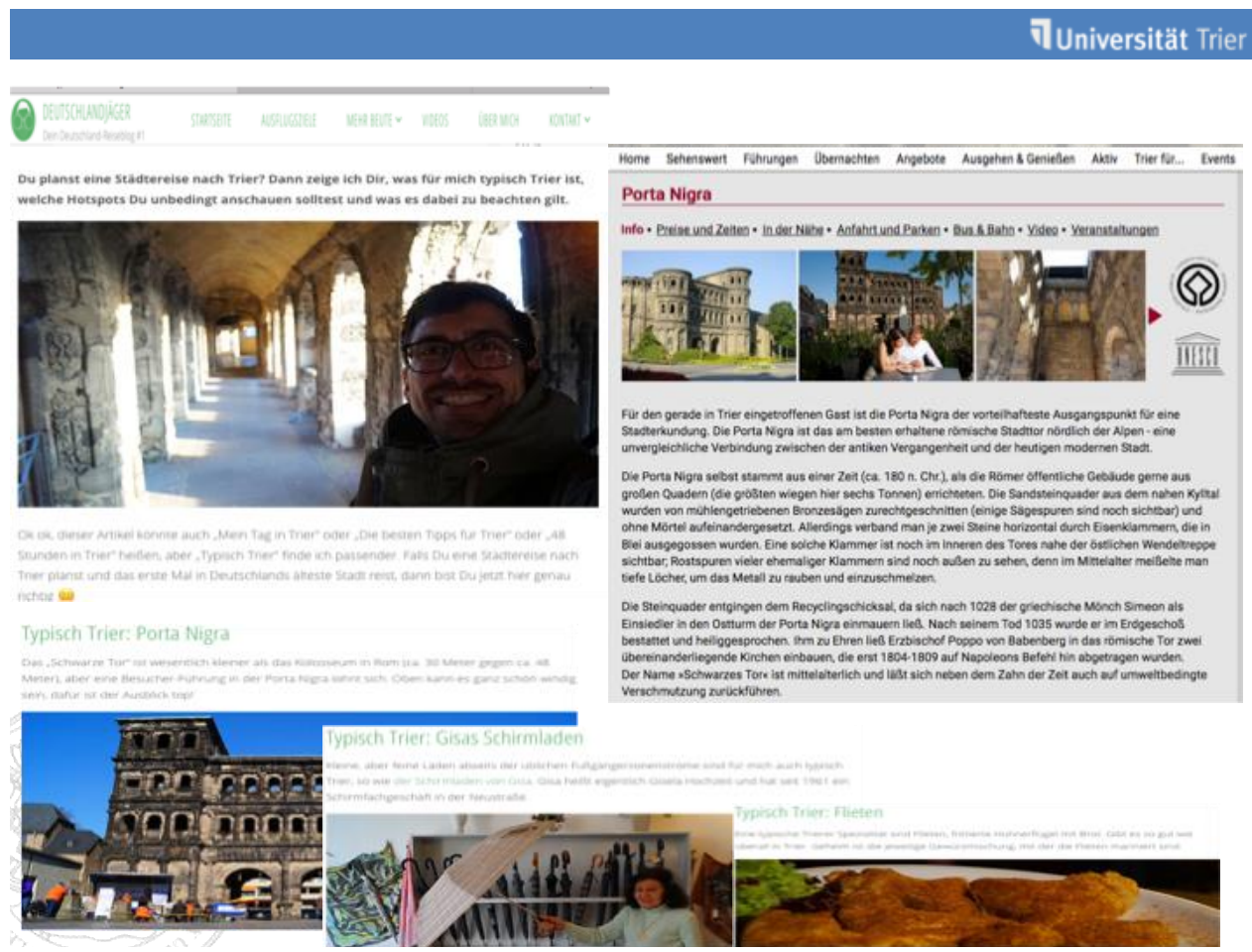

Reiseblog: Deutschlandjäger. Dein Deutschland-Reiseblog \# 1: http://deutschlandjaeger.de/ (zuletzt aufgerufen: 10.06.2018)

Trier Tourismus und Marketing: http://www.trier-info.de/portanigra-info (zuletzt aufgerufen 15.11.2016)

Entscheidender Unterschied zwischen Reiseblog und Stadt-Webseite sind aber die Art der Darstellung und Perspektive auf das Reiseziel wieauch die Beziehung zwischen Schreiber/Blogger und dem potentiellen Leser, was nun näher betrachtet werden soll:

\section{Reiseblog „Deutschlandjäger. Dein Deutschland-Reiseblog \# 1“}

Der Reiseblog „Deutschlandjäger“ ist ein privater Blog. Er wird von Jan betrieben, der sich in der Rubrik ,über mich“ „als Deutschlandjäger“ vorstellt, als jemand, der ,gern reist und als Reiseblogger am liebsten durch Deutschland [zieht]“, „, immer auf der Jagd nach den schönsten Ecken in Deutschland“" ist,,,grundsätzlich [s]eine persönliche Meinung darstellt“_sich 


\section{TEXTSORTENBASIERTE SPRACHARBEIT}

über „Rückmeldungen jeglicher Art freut“ und den Besuchern vor allem zeigen möchte, „wie toll das eigene Land ist“ ${ }^{13}$. Der hier verwendeten Jagdmetapher kommt dabei eine Art „blogstiftende bzw. -rahmende“ Funktion zu, neben Deutschlandjäger werden die weiteren Ziele unter „Mehr Beute“ (vgl. Menüleiste) aufgeführtundder gewöhnlich mit „weiterlesen“ versehene Button hier mit weiterjagen bezeichnet.

Der Blogger beschreibt seinen Blick auf die Stadt, wählt das aus, was er als kulturell bedeutsam, charakteristisch und lohnenswert empfindet: Das sind Sehenswürdigkeiten, wie die Porta Nigra, die Kaiserthermen oder das Rheinische Landesmuseum, aber auch regionale Spezialitäten und ihre entsprechenden regionalen Bezeichnungen, wie die Flieten (frittierte Hühnerflügel, die in einer bestimmten Gewürzmischung mariniert werden) oder besondere „Objekte“, wie der Schirmladen von Gisa. Er sieht sich als „Jäger", seine Einträge sind eine Mischung aus kleinen Texten und dazu passenden Fotos oder Videos, verknüpft mit vielen Erfahrungen, Tipps und Empfehlungen zum Reiseziel - alles ist gekennzeichnet durch die IchPerspektive und damit durch ein hohes Maß an Subjektivität, eine große Nähe zum Leser, verbunden mit dem Wecken positiver Emotionen, Gefühle und Begeisterung.

Sprachlich werden Subjektivität, Nähe zum Leser und Emotionalität realisiert durch eine Anzahl von Merkmalen, die man der NäheKommunikation, die eher konzeptionell mündlich ist, zurechnen kann. Linguistisch lassen sich diese Phänomene gut auf der Folie von Nah- und Distanzkommunikation, wie es in dem bekannten Modell von Koch/Oesterreicher (1994) dargelegt wurde, beschreiben. So fällt für den gesamten Blog auf, dass die persönliche Du-Anrede verwendet wird und oft auf das dialogische Prinzip und rhetorische Fragen zurückgegriffen wird, wie z.B.

„Ok ok, dieser Artikel könnte auch `Mein Tag in Trier` oder 'Die besten Tipps für Trier` oder `48 Stunden in Trier' heißen, aber 'Typisch Trier' finde ich passender. Falls Du eine Städtereise nach Trier planst und das erste Mal in Deutschlands älteste Stadt reist, dann bist Du jetzt hier genau richtig"[...]

\footnotetext{
${ }^{13} \mathrm{Vgl}$. https://deutschlandjaeger.de/ueber-diesen-reiseblogger/.
} 
„Du planst eine Städtereise nach Trier? Dann zeige ich Dir, was für mich typisch Trier ist, welche Hotspots Du unbedingt anschauen solltest und was es dabei zu beachten gilt.“ 14

Nähe zum Leser und Emotionalität als Momente sprechsprachlicher Kommunikation kann man auch in den einzelnen Beschreibungen zu den ausgewählten Sehenswürdigkeiten finden, wobei neben geringeren deskriptiven Anteilen (geschichtliche wie auch kulturhistorische Fakten) besonders die instruierenden und evaluativ-empfehlenden Passagen auffallen, in denen persönliche Eindrücke, Begegnungen und Erfahrungen des Bloggers emotional weitergegeben werden, wie z.B.

„Das `Schwarze Tor’ ist wesentlich kleiner als das Kolosseum in Rom (ca. 30 Meter gegen ca. 48 Meter), aber eine BesucherFührung in der Porta Nigra lohnt sich. Oben kann es ganz schön windig sein, dafür ist der Ausblick top!“ [...]

„Die Porta Nigra ist das Wahrzeichen Triers und bildet ein Ende der Altstadt. Abends sind nicht mehr so viele Leute auf dem Vorplatz, dann bleibt Dir genug Raum und Zeit für ein paar schöne Nachtaufnahmen der Porta. “"15

Als evaluativ-empfehlende sprachliche Mittel findet man im Blog zu Trier neben unmarkierten bewertenden Lexemen wie etwas lohnt sich, etwas sollte man unbedingt anschauenin der Region bekannte bildliche Ausdrücke wie Typisch Trier, Typisch Porta oder Trier - das größte Dorf der Eifel viele umgangssprachlicheAusdrücke bzw. Phrasen wie etwas ist top, Hotspots, ganz schön windig, richtig gut ist, dass ..., besonders witzig finde ich ..., ziemlich schade finde ich ..., ich muss ja zugeben, etwas ist nicht mein Ding,die emotional verstärkende Wirkung haben.

Nähe-Kommunikation und damit den Transfer gesprochen-sprachlicher Formen ins Schriftliche kann man auch auf der syntaktischen Ebene erkennen, so werden einfachere Satzstrukturen, Ellipsen (wie im Beispiel Tilgung des formalen Subjektpronomens im 3. Satz) und Interjektionen verwendet, wie die folgenden Beispiele zeigen:

„Der Trierer Dom gilt als die älteste Kirche in Deutschland. Leider kannst Du nicht nach oben, was ich ziemlich schade finde. Gibt nämlich keine Aussichtsplattform oder ähnliches.

\footnotetext{
${ }^{14} \mathrm{https}: / /$ deutschlandjaeger.de/?s=Trier.

${ }^{15}$ https://deutschlandjaeger.de/typisch-trier/. 


\section{TEXTSORTENBASIERTE SPRACHARBEIT}

Drinnen ist es aber auch schön. [...] Aha. Die Heilige Pforte ist $[\ldots]^{616}$

Weitere Tilgungen als Transferphänomene aus der gesprochenen Sprache finden sich im Blog z.B. beim unbestimmten Artikel wie Die Barbarathermen sind ne Art Zweigstelle der Kaiserthermen oder Dafür gibt es im sogenannten 'größten Dorf der Eifel ne Menge zu sehen ${ }^{17}$. Wie in linguistischen Analysen $\mathrm{zu}$ Weblogs schon beschrieben (vgl. bes. Schlobinski/Siever 2005), wird bei der Schreibung der verkürzten Formen zumeist auf den Apostroph verzichtet.

An einigen wenigen Stellen findet man im Blog auch Inflektive, ,prädikativ gebrauchte Verbstämme“ (Schlobinski/Siever 2005: 77), wie man sie aus der Chat-Kommunikation zum Ausdruck von Gefühlen bzw. Handlungen kennt, um das schriftlich auszudrücken, was der jeweils andere nicht wahrnehmen kann. Im Blogbeispiel Landesmuseum = Gähn? wird das Museum mit Gähnen gleichgesetzt, damit eine oft bei jüngeren Leuten zu beobachtende ablehnende Einstellung gegenüber Museen aufgegriffen, um sie aber durch das Fragezeichen wie auch die im Anschluss ausgedrückte persönliche Erfahrung zugleich zu revidieren und sogar ins Gegenteil (auch mit dem Wortspiel) zu verkehren.

„Landesmuseum $=$ Gähn? Ich muss ja zugeben, dass Heimatund Landesmuseen nicht unbedingt mein Ding sind, aber im Landesmuseum Trier ist es irgendwie anders: 'Das ist ein Römer, Schatz! ‘“18

Aufmerksam gemacht soll auch auf die verwendeten Smileys werden, die ebenfalls als Ausdruck von Emotionen und Gefühlen gebraucht werden, wie auch Nähe zwischen den Kommunikationspartnern schaffen.

So ist der Blog insgesamt ein Beispiel für eine aktuelle Kommunikationsform, ,in der Mündlichkeit und Schriftlichkeit“, wie es Schlobinski/Siever formulieren, ,zu einer spezifischen hybridisierten Praxis verschmelzen“" (ebd.: 82).

\footnotetext{
${ }^{16} \mathrm{https}: / /$ deutschlandjaeger.de/typisch-trier/.

${ }^{17} \mathrm{https}: / /$ deutschlandjaeger.de/typisch-trier/.

${ }^{18}$ https://deutschlandjaeger.de/?s=Trier.
} 


\section{Webseite der Stadt Trier}

Auch die Webseite der Stadt Trier, die von der Trier Tourismus und Marketing $\mathrm{GmbH}$ betrieben wird, will bei potentiellen Reisenden Interesse für Trier wecken, eine positive Grundhaltung dem Reiseziel gegenüber erreichen, indem sie über das Reiseziel Trier - wie an den Rubriken auf der Menüleiste zu erkennen ist ${ }^{19}$ - informiert und instruiert, also Informationen und Empfehlungen für die Gestaltung eines Aufenthaltes in der Stadt und Umgebung gibt. Der abgebildete Eintrag über die Porta Nigra ${ }^{20}$ findet sich unter „Sehenswert“ und ist Teil der Rubrik „Römerbauten“, für die Trier als älteste Stadt Deutschlands sehr bekannt ist.

Im Gegensatz zum Reiseblog zeichnet sich die Webseite durch einen größeren Faktenreichtum aus. So werden Informationen $\mathrm{zu}$ vielen kulturhistorisch bedeutsamen Bauwerken, wie Römerbauten, Kirchen, Schlössern, Denkmälern aber auch Ausflugszielen in der Umgebung, verbunden mit Informationen zu Öffnungszeiten, Anfahrtswegen, aktuellen Veranstaltungen etc., gegeben. Die Perspektive auf das Reiseziel ist eher objektiv, geworben und in besonderer Weise aufmerksam gemacht wird mithilfe der Einzigartigkeit und Bedeutsamkeit einzelner Objekte. Die Beziehung zwischen Webseite und Leser ist distanzierter. Sprachlich realisiert wird diese Kommunikationsweise v.a. durch anschauliche und faktenreiche Beschreibungen der einzelnen Sehenswürdigkeitenund einer eher indirekten Anrede potentieller Besucher.

So sind die Beschreibungen zu den einzelnen Sehenswürdigkeiten sehr genau, detailliert und am einzelnen Objekt orientiert. Es werden ausführliche Informationen zur Struktur und Entstehung der einzelnen Bauwerke wie zu ihrer kulturhistorischen Bedeutung und Funktion, verknüpft mit näheren Erkundungs- und aktuellen Besichtigungshinweisen gegeben, wie das Beispiel zeigt:

„Für den gerade in Trier eingetroffenen Gast ist die Porta Nigra der vorteilhafteste Ausgangspunkt für eine Stadterkundung. Die Porta Nigra ist das am besten erhaltene römische Stadttor nördlich der Alpen. [...] Die Porta Nigra selbst stammt aus einer Zeit (ca. 170 n. Chr.), als die Römer öffentliche Gebäude gerne aus großen Quadern (die größten wiegen hier 7 Tonnen) errichteten. Die Sandsteinquader aus dem nahen Kyltal wurden von mühlengetriebenen Bronzesägen zurechtgeschnitten (einige Sägespuren sind noch sichtbar) und ohne Mörtel

${ }^{19}$ https://www.trier-info.de/sehenswert-in-trier.

${ }^{20}$ https://www.trier-info.de/portanigra-info. 
aufeinandergesetzt. [...] Im Inneren sind Spuren der Doppelkirche, römische Steinmetzzeichen und Datumsgraffiti $\mathrm{zu}$ sehen; die Aussichten auf Stadt und Tal sind bemerkenswert. “21

Sprachlich begegnet man einer v.a. deskriptiven Themenentfaltung, charakteristisch ist eine gehäufte Verwendung von Adjektivattributen, adverbialen Attributen, Superlativen und einem stark thematischen, teilweise fachsprachlich geprägten Wortschatz (Komposita, Verben). Mithilfe der Attributewie öffentliche Gebäude, römisches Stadttor, große Quader, mühlengetriebene Bronzesägen, Sandsteinquader aus dem nahen

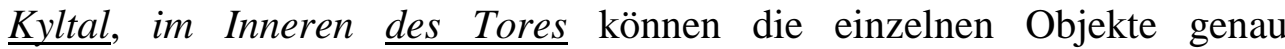
charakterisiert und spezifisch beschrieben werden. Wie Fandrych/Thurmair schreiben, tragen sie häufig in verdichteter Form den Kern der Aussage und sind kommunikativ notwendig (Fandrych/Thurmair 2011b: 68). Die Superlative wie der vorteilhafteste Ausgangspunk oder das am besten erhaltene römische Stadttor unterstreichen nicht nur das Besondere und Einzigartige, sondern drücken auch Bewertung bzw. Empfehlung aus.

Eine wesentliche Rolle bei der Beschreibung der einzelnen Sehenswürdigkeiten kommt dem thematischen Wortschatz zu. So kann man im ausgewählten Beispiel ein kultursensitives Sach- bzw. Wortfeld ausmachen, das Wörter mit enzyklopädischen Aspekten (Kultur-, Bau- und Kirchengeschichte) wie auch soziokulturelle und fachsprachlich geprägte Lexeme zusammenordnet, wie Quader, Sandsteinquader, Bronzesäge, Mörtel, Eisenklammer, Blei, Wendeltreppe, Doppelkirche, Einsiedler, Steinmetzzeichen, aufeinandersetzen, gießen, meißeln, schmelzen. Zur genauen Charakterisierung werden, wie die Beispiele Bronzesäge, Sandsteinquader oder Eisenklammer zeigen, auch Determinativkomposita genutzt. Dem thematischen Wortschatz sind im weitesten Sinne auch die regional-landeskundlichen Lexeme, wie geographische Benennungen Kyltal (die Kyl ist ein kleiner Fluss in der Eifel) oder Personennamen Erzbischof Poppo von Babenberg, Mönch Simeon, zuzurechnen, die zum Verorten der Objekte in Raum und Zeit notwendig sind.

Während persönliche Empfehlungen und Erfahrungen für den Reiseblog ein dominierender Textbaustein bzw. charakteristisches Textmuster sind, begegnet man ihnen auf der Webseite eher selten. Und wenn bewertende Passagen, wie z.B. die Aussichten auf Stadt und Tal sind bemerkenswert,

${ }^{21}$ https://www.trier-info.de/portanigra-info. 
vorteilhaftester Ausgangspunkt oder grandios der Blick vom barocken St. Georgsbrunnen [...] ein Platz zum Verweilen, Entspannen und Nachdenken zu lesen sind, dann werden lexikalische Mittel genutzt, bei denen auch empfehlende Bedeutung mitschwingt.

Schließlich noch ein Blick auf die Gestaltung der Beziehung zum Leser bzw. potentiellen Reisenden. Wird im Reiseblog die Du-Anrede als charakteristisches Merkmal verwendet, so begegnet man auf der Webseite meist unpersönlichen Formen wie für den gerade in Trier eingetroffenen Gast, die Zuschauer, der Besucher. An einigen Stellen, in denen auf aktuelle Besichtigungen hingewiesen und für diese geworben wird, findet man auch die direkte Sie-Anrede, wie ein römischer Zenturio zeigt Ihnen das Geheimnis der Porta Nigra. Mit den unpersönlichen Formen wird eher Allgemeingültigkeit ausgedrückt.

Methodisch-didaktische Schlussfolgerungen sind für die Behandlung dieser beiden Textsorten in mehrfacher Hinsicht zu ziehen. So ist es aus rezeptionsdidaktischer Sicht unabdingbar, den kommunikativpragmatischen Rahmen beider Texte zu vermitteln. Die Lernenden müssen für die Perspektive der Schreibenden und die damit verbundene sprachliche Realisierung sensibilisiert werden. Fragen, wie die folgenden, können dabei helfen: Wo kann man die Webseite bzw. den Blog finden?, Wer hat sie/ihn geschrieben?, An wen richtet sich die Webseite oder der Blog?, Welche Absicht verfolgt die Webseite/der Blog? Die Antworten auf diese Fragen ermöglichen den Lernenden ein globales Verstehen der Texte, zugleich müssen sie Absichten, Wertungen und Einschätzungen der Schreibenden (Textproduzenten) reflektieren. Auf diese Weise können beide Texte pragmatisch situiert werden. Hinsichtlich der Förderung produktiver Fertigkeiten sind besonders die ergiebigen Transfers auf persönliches Schreiben und Sprechen (Beschreiben der eigenen Stadt/Lieblingsstadt, Empfehlen einer besonderen Sehenswürdigkeit etc.) und der damit verbundene Auf- und Ausbau eines thematisch-usuellen Wortschatzes wie auch entsprechendeFormulierungen hervorzuheben. So lässt sich der textbasierte Wortschatz für eine an gedächtnis-und lernpsychologisch ausgerichteten Erkenntnissen orientierte textbezogene Wortschatzarbeit nutzen, indem neben den angesprochenen Sach- bzw. Wortfeldern weitere Beziehungen der Wörter im Text aufgezeigt werden, wie beispielsweise syntagmatische Relationen, so typische Adjektiv-Substantiv-Verbindungen (öffentliche/historische/mittelalterliche Gebäude, römisches/ mittelalterliches Stadttor, ereignisreiche Zeit) oder usuelle präpositionale 


\section{TEXTSORTENBASIERTE SPRACHARBEIT}

Fügungen wie Platz zum Verweilen/Entspannen/Nachdenken oder paradigmatische Beziehungen wie Quader und Sandsteinquader.

\section{Texte als Vertreter von Textsorten: Ein Blick in aktuelle Lehrwerke}

In der folgenden stichprobenartigen Analyse soll ermittelt werden, in wieweit es den Lehrwerken gelingt, bei der Arbeit mit Texten diese als Vertreter einer Textsorte wahrzunehmen undihre entsprechenden Merkmale für die Ausbildung textueller Kompetenzen zu nutzen. Für die Stichprobe ausgewählt wurden folgende Lehrwerke der Grundstufe A1-B1: „studio d“, „Menschen“ und „DaF kompakt" ${ }^{\text {"22 }}$. Diese Lehrwerke wenden sich an Erwachsene und junge Erwachsene, werden in Sprachkursen an der Universität Trier wie auch im Ausland, so z.B. an unserer spanischen Partneruniversität in Valencia, verwendet. In allen drei Lehrwerken sind Texte wesentlicher Input, in einigen wird explizit auf Textsorten verwiesen. So formuliert das Vorwort $\mathrm{zu}$,studio d“: „Durch handlungs- und inhaltsbezogene Aufgaben gelingt es den Lernenden rasch, kompetent mit unterschiedlichen Textsorten aus Alltag, Beruf, Kultur und Freizeit umzugehen“ (studio d 2006: 1) und „Menschen A1“: „Gezieltes Fertigkeitstraining, das unterschiedliche authentische Textsorten [...] umfasst" (Menschen 2012: 3).

Nachgespürt werden soll besonders den Aufgaben, die das Verstehen vorbereiten und steuern, denn die gewählten Textsorten stellen differenzierte Anforderungen an das Lese- bzw. Hörverstehen. Im Zusammenhang damit wird auch die textbasierte Arbeit am Wortschatz betrachtet.

\section{Wetterberichte}

In allen drei Lehrwerken spielt das Thema Wetter eine Rolle und ist mit sinnvollen Lernzielen bzw. Sprachhandlungen des Alltags, wie „Wetterinformationen verstehen, über Wetter sprechen“ (studio d 2005: 7), „Wetterkarte und Wetterbericht verstehen“ (DaF kompakt 2011: 6) bzw. „über das Wetter sprechen“ (Menschen 2012: 4) verknüpft. Dementsprechend wird unter den Lernzielen zum Wortschatz in den Lehrwerken auch explizit auf das Wortfeld „Wetter“ verwiesen (vgl. ebd.).

${ }^{22}$ Die genauen Literaturangaben zu den Lehrwerken erscheinen im Literaturverzeichnis. 
Das Verstehen von Wetterinformationen in Form von Wetterberichten wird aber nur in den Lehrwerken „Menschen“ und „DaF kompakt" thematisiert: So sollen die Lernenden z.B. einzelne Informationen über das Wetter, wie Temperaturangaben, heraushören (vgl. Menschen 2012: 85) oder Symbole auf einer Wetterkarte den entsprechenden Wetterberichten zuordnen (vgl. DaF kompakt 2011: 116). Positiv fällt dabei auf, dass die Texte als authentisch angesehen werden können und auch die Hör- bzw. Leseaufträge Anforderungen simulieren, die im Alltag mit dem Verstehen von Wetterberichten verbunden sind. Probleme dürften die Aufgaben aber dennoch bereiten, weil die Lernenden auf das angestrebteVerstehensziel nicht ausreichend vorbereitet werden. Es gibt zwar eine thematischlexikalische Einbettung des Textes innerhalb der Lektionen, eine konzeptgeleitete Vorentlastung und Steuerung ${ }^{23}$, die textsortenspezifische Elemente wie Gliederungen oder usuelle Wortverbindungennutzt, erfolgt aber nur ansatzweise.

Dem in den Wetterberichten verwendeten Wortschatz wird in beiden Lehrwerken unterschiedliches Gewicht beigemessen. So fokussiert „DaF kompakt" auf rezeptive Kompetenzen und führt den Grundwortschatz situationsdifferenziert über ein Alltagsgespräch und eine Wetterkarte ein, erfasst dadurch geschickt gebräuchliche Nomen, Verben undAdjektive, wie sie in einem Alltagsgespräch über Wetter und in einem Wetterbericht vorkommen, so z.B. neblig, sonnig aber auch stark bewölkt, meist regnerisch, vereinzelt Gewitter, meistens heiter. Kritisch festzuhalten bleibt allerdings, dass dieser Wortschatz für die Lernenden nicht weiter aufbereitet wird, wirkliche Wortschatzaufgaben sucht man vergeblich.

Im Lehrwerk „Menschen“ wird das neue Wortfeld in jeder Lektion als Bildlexikon präsentiert (vgl. Menschen 2012: 6 bzw. 62), ein Ansatz, der den visuellen Kanal zur Semantisierung und Memorierung ergänzend nutzt. Mithilfe der Bilder und einfacher Beschreibungen entsteht ein Wortfeld zum Thema Wetter, das am Lexikon orientiert ist, v.a. Nomen und davon abgeleitete Verben wie auch Adjektive präsentiert, damit den Wortschatz aber eher isolierend einführt. Dem textbasierten Wortschatz nämlich neben dem Wetterbericht gibt es auch Auszüge aus einem Urlaubswetterblog - wird im Lehrwerk keinerlei Beachtung geschenkt. Bedenkt man aber, dass charakteristischen Wörtern und Wendungen eine Signalfunktion für das Verstehen (Aufbau, Gliederung und Struktur von Text(sort)en) zugesprochen wird, dann kommt der Rezeption und damit

${ }^{23}$ zur Verstehensdidaktik vgl. v.a. Solmecke 


\section{TEXTSORTENBASIERTE SPRACHARBEIT}

dem Verstehen und Erklären von Wörtern und Formulierungen aus Texten entsprechende Bedeutung zu. Im Lehrwerk aber fehlen Aufgaben und Übungen, die diesen Textwortschatz bzw. Textsortenwortschatz wieder aufnehmen, ihn unter verschiedenen Aspekten neu ordnen, auch mit dem eingeführten Wortfeld verknüpfen, erweitern und anwendbar machen.

\section{Städte-Webseiten und Reiseblog}

Das Thema „Reisen“ spielt in allen drei Lehrwerken eine Rolle, der damit verknüpfte textliche Input ist aber unterschiedlich. Reiseführertexte bzw. Städte-Webseiten und Einträge in Reiseblogs kommen auf unterschiedlichen Niveaustufen (A2 und B1) mehrfach in „DaF kompakt" vor, wie z. B. in der Lektion 15 „In Wien unterwegs“, in Lektion 21 „Kreativ in Hamburg“ oder in Lektion 28 „Innsbruck, die Hauptstadt von Tirol“ (DaF kompakt 2011: 128f, 172f und 232). Positiv fällt hierbei auf, dass die Texte bzw. Textausschnitte eine gewisse didaktische Authentizität erkennen lassen, also wesentliche Textsortenmerkmale tragen. Und auch das mit den Texten verbundene primäre Leseziel „Informationen über Sehenswürdigkeiten in Wien/Hamburg/Innsbruck verstehen“ bzw. „Eindrücke über eine Stadt verstehen" (ebd.: 6ff) ist an den natürlichen Verwendungsbedingungen dieser Textsorten orientiert. Gelungen ist auch die Verbindung von Reiseführertext bzw. Stadt-Webseite und persönlichem Reiseblog, denn dadurch werden dem Lerner verschiedene Perspektiven auf die ausgewählten Ziele präsentiert.

Betrachtet man aber die das Verstehen vorbereitenden und steuernden Aufgaben, so wird deutlich, dass sie die Textsortenmerkmale kaum berücksichtigen, es damit keinen Bezug zur Textsorte gibt, die Textsorte nicht zum Lerngegenstand wird. Dadurch gehen die kommunikativpragmatischen Aspekte verloren. Die Perspektiven der Schreiber (Reiseführer/Stadt-Webseite vers. persönlicher Blogeintrag), ihre Absichten, Wertungen und Einschätzungen, die für eine adäquate Rezeption aber unerlässlich sind, bleiben unbeachtet. Dagegen konzentrieren sich die Aufgaben lediglich auf den Textinhalt bzw. auf die in den Texten angesprochenen Fakten. Beliebte methodisch-didaktische Umsetzungen hierfür sind Foto-Text-Zuordnungen und W-Fragen, wie beispielsweise „Welcher Text passt zu welchem Foto? Notieren Sie.“ (ebd.: 128) oder „Überfliegen Sie die Texte aus einem Reiseführer über Hamburg. Welches 
Foto oben passt zu welchem Text? Lesen Sie die Texte noch einmal und beantworten Sie die Fragen“" (ebd.: 172f).

Für eine adäquate Rezeption spielen lexikalische Mittel und entsprechende Formulierungsweisen wie auch die Gestaltung des Bezugs zwischen Schreiber und Leser eine wesentliche Rolle. Dem Text- bzw. Textsortenwortschatz sollte somit als konstituierendes Merkmal Aufmerksamkeit geschenkt werden. Aufgaben zum Wortschatz aber sucht man im Lehrwerk vergeblich.

Fasst man die Befunde zusammen, so lassen sich folgende Schlussfolgerungen formulieren: Positiv zu bewerten ist in den Lehrwerken „DaF kompakt“ und „Menschen“ die Qualität der Texte, die als authentisch bzw. semiauthentisch angesehen werden können. Problematisch bleibt aber, dass die in den Lehrwerken verwendeten Texte nicht als Vertreter einer Textsorte wahrgenommen werden und ihre entsprechenden Merkmale für die Ausbildung textueller Kompetenzen ungenutzt bleiben. Bei der Textarbeit dominiert noch immer eine stark auf inhaltliche Aspekte hin ausgerichtete Arbeitsweise und damit ein verengender Blick auf Texte, der sie ihre Textsortenqualität einbüßen lässt und Lernende kaum in die Lage versetzt, mit Texten kompetent umzugehen, sie letztlich für den eigenen Spracherwerb zu nutzen. Weitere Anstrengungen sind also vonnöten.

\section{Literatur}

Adamzik, Kirsten 2011: Textsortennetze. In: Habscheid, Stefan (Hrsg.): Textsorten, Handlungsmuster, Oberflächen: Linguistische Typologien der Kommunikation. New York, 367-389.

Bachmann, Thomas/Feilke, Helmuth 2014 (Hrsg.): Werkzeuge des Schreibens. Beiträge zu einer Didaktik der Textprozeduren. Stuttgart: Fillibach bei Klett.

Bausch, Richard u.a. 2007 (Hrsg.): Textkompetenzen. Arbeitspapiere der 27. Frühjahrskonferenz zur Erforschung des Fremdsprachenunterrichts. Tübingen.

Beese, Melanie/Roll, Heike 2015: Textsorten im Fach - zur Förderung von Literalität im Sachfach in Schule und Lehrerbildung. In: Benholz, Claudia/Frank, Magnus/Gürsoy, Erkan (Hrsg.): Deutsch als Zweitsprache in allen Fächern. Konzepte für Lehrerbildung und Unterricht. Stuttgart, 51-73. 
Benholz, Claudia/Frank, Magnus/Gürsoy, Erkan 2015 (Hrsg.): Deutsch als Zweitsprache in allen Fächern. Konzepte für Lehrerbildung und Unterricht. Stuttgart: Fillibach bei Klett.

Bittner, Jürgen 2003: Digitalität, Sprache, Kommunikation. Eine Untersuchung zur Medialität von digitalen Kommunikationsformen und Textsorten und deren variationslinguistischer Modellierung. Berlin: Erich Schmidt Verlag.

Brinker, Klaus 2010: Linguistische Textanalyse. Eine Einführung in Grundbegriffe und Methoden. Berlin.

Decke-Cornill, Helene/Küster, Lutz 2010: Fremdsprachendidaktik. Tübingen: Narr Verlag.

Fandrych, Christian 2010: Grammatikerwerb und Grammatikvermittlung. In: Krumm, Hans-Jürgen u.a. (Hrsg.): Deutsch als Fremd- und Zweitsprache. Ein internationales Handbuch. 2 Bde. Berlin/ NewYork: de Gruyter [=HSK - Handbücher zur Sprach- und Kommunikationswissenschaft, Bd. 35], 1008-1021.

Fandrych, Christian/Thurmair, Maria 2011a: Textsorten im Deutschen. Linguistische Analysen aus sprachdidaktischer Sicht. Tübingen.

Fandrych, Christian/Thurmair, Maria 2011b: Plädoyer für eine textsortenbezogene Sprachdidaktik. In: Zeitschrift DaF 02/11, 84-94.

Feld-Knapp, Ilona 2005: Textsortenspezifische Merkmale und ihre Relevanz für Spracherwerbsprozesse (DaF). In: Adamzik, Kirsten/Krause, Wolf-Dieter (Hrsg.): Text-Arbeiten. Textsorten im fremd- und muttersprachlichen Unterricht an Schule und Hochschule. Tübingen, 111-130.

Freudenberg-Findeisen, Renate 2013: Netze-Texte-Felder. Für eine text(sorten)fundierte Grammatikarbeit. In: Fernández Bueno, M./Llamas Ubieto, M./Sánchez Hernández, P. (Hrsg.): Rückblicke und neue Perspektiven - Miradas retrospectivas y nueva sorientaciones. Frankfurt/M.: Peter Lang Verlag, 2013, 351-363.

Freudenberg-Findeisen, Renate 2014: Verschenkte Möglichkeiten: Zur Arbeit mit Textsorten in DaF-Lehrwerken. In: Shchipitsina, Ludmilla (Hrsg.): Subjekt der Kognition und Kommunikation: Sprache und Kultur: Festschrift für Ludmilla Grischaewa. Woronesch 2014, 564591.

Freudenberg-Findeisen, Renate 2016 (Hrsg.): Auf dem Weg zu einer Textsortendidaktik. Linguistische Analysen und text(sorten)didaktische Bausteine nicht nur für den fremdsprachlichen Deutschunterricht. Hildesheim: Olms. 
Hallet, Wolfgang 2010: Umgang mit Texten und Medien. In: Hallet, Wolfgang/Königs, Frank G. (Hrsg.): Handbuch Fremdsprachendidaktik. Seelze-Velber: Kallmeyer in Verbindung mit Klett.

Heinemann, Wolfgang 2000: Textsorte - Textmuster - Texttyp. In: Brinker, Klaus/Antos, Gerd/ Heinemann, Wolfgang/Sager, Sven (Hrsg.): Text- und Gesprächslinguistik. HSK-Bd. 16.1. Berlin/New York, 507-523.

Heinemann, Wolfgang/Heinemann, Margot 2002: Grundlagen der Textlinguistik. Tübingen.

Hufeisen, Britta 2008: Textsortenwissen - Textmusterwissen Kulturspezifik von Textsorten. In: Fremdsprache Deutsch, 39, 50-55.

Koch, Peter/Oesterreicher, Wulf 1994: Schriftlichkeit und Sprache. In: Günther, Hartmut/Ludwig, Otto (Hrsg.): Schrift und Schriftlichkeit. Writing and Its Use. Ein interdisziplinäres Handbuch internationaler Forschung. 1.Halbbd., Berlin, New York (Handbücher zur Sprachund Kommunikationswissenschaft 10.1), S. 587-604.

Krames, Marion 2016: Von der Wortschatzwende zur Textsortenwende? Wertende lexikalische Mittel in Lehrbüchern und Internettexten. In: Freudenberg-Findeisen, Renate (Hrsg.): Auf dem Weg zu einer Textsortendidaktik. Linguistische Analysen und text(sorten)didaktische Bausteine nicht nur für den fremdsprachlichen Deutschunterricht. Hildesheim, 219-241.

Kühn, Peter 2013: Wortschatz. In: Oomen-Welke, Ingelore/Ahrenholz, Bernt (Hrsg.) Deutsch als Fremdsprache. Baltmannsweiler: Schneider Verlag Hohengehren, 153-165.

Neuland, Eva 2006 (Hrsg.): Variation im heutigen Deutsch: Perspektiven für den Unterricht. Frankfurt/M: Peter Lang Verlag.

Neuland, Eva/Peschel, Corinna 2013: Einführung in die Sprachdidaktik. Stuttgart/Weimar.

Portmann-Tselikas, Paul R. 2000: Der Einfluss der Textlinguistik auf die Fremdsprachendidaktik. In: Brinker, Klaus u.a. (Hrsg.). Text- und Gesprächslinguistik. Ein internationales Handbuch zeitgenössischer Forschung. 1. Halbband. Berlin/New York: de Gruyter, 830-842.

Portmann-Tselikas, Paul R./Schmölzer-Eibinger, Sabine 2008: Textkompetenz. In: Fremdsprache Deutsch, 39, 5-17.

Schlobinski, Peter/Siever, Torsten 2005 (Hrsg.): Sprachliche und textuelle Merkmale in Weblogs. NET.WORX 46 http://www.mediensprache.net/networx/ 
Solmecke, Gert 1993: Texte hören, lesen und verstehen. München: Langenscheidt.

Spiekermann, Helmut 2010: Variation in der deutschen Sprache. In: Krumm, Hans-Jürgen u.a. (Hrsg.): Deutsch als Fremd- und Zweitsprache. Ein internationales Handbuch. 2 Bde. (=HSK Handbücher zur Sprach- und Kommunikationswissenschaft, Bd. 35),Berlin/ New York: de Gruyter, 343-360.

Spillner, Bernd 1983: Zur kontrastiven Analyse von Fachtexten - am Beispiel der Syntax von Wetterberichten. In: Zeitschrift für Literaturwissenschaft und Linguistik, 51/52, 110-123.

Stein, Stephan 2004: Texte, Textsorten und Textvernetzung. Über den Nutzen der Textlinguistik (nicht nur) für die Fremdsprachendidaktik. In: Lüger, Heinz-Helmut/Rothenhäusler, Rainer (Hrsg.): Linguistik für die Fremdsprache Deutsch. Landau, 171-222.

Stein, Stephan 2016: Anwendungsperspektiven für die Textsortenlinguistik: Der Nutzen mehrdimensionaler bzw. holistischer Textsortenanalysen. In: Freudenberg-Findeisen, Renate (Hrsg.): Auf dem Weg zu einer Textsortendidaktik. Linguistische Analysen und text(sorten)didaktische Bausteine nicht nur für den fremdsprachlichen Deutschunterricht. Hildesheim, 27-47.

Steinhoff, Torsten/Sorrentino, Daniela 2010: Textwortschatzerwerb im Sprachvergleich. In: Foschi, A.M./ Hepp, Marianne/Neuland, Eva/Dalmas, Martine (Hrsg.): Text und Stil im Kulturvergleich. Pisaner Fachtagaung 2009 zu interkulturellen Wegen Germanistischer Kooperation. München: iudicium Verlag, 192-215.

Thonhauser, Ingo 2010: Textarbeit. In: Krumm, Hans-Jürgen u.a. (Hrsg.): Deutsch als Fremd- und Zweitsprache: ein internationales Handbuch (=Handbücher zur Sprach- und Kommunikationswissenschaft; Bd. 35) Berlin/New York: de Gruyter, 1033-1039.

Venohr, Elisabeth 2007: Textmuster und Textsortenwissen aus der Sicht des Deutschen als Fremdsprache. Textdidaktische Aspekte ausgewählter Textsorten im Vergleich Deutsch-Französisch-Russisch. Frankfurt am Main.

Zhang, Wei 2011: Einfluss der sprachsystematischen Bedingtheit auf die kulturspezifische Textgestaltungam Beispiel deutscher und chinesischer Kontaktanzeigen. In: Sprachreport 2/2011, 32- 35.

\section{Lehrwerke}


Aspekte:

Koithan, Ute/Schmitz, Helene u. a. (20006): Aspekte. Mittelstufe Deutsch B1+. München: Langenscheidt.

DaF kompakt:

Sander, Ilse/Braun, Birgit/Doubek, Margit u. a. 2011: DaF kompakt A1 - B1. Deutsch als Fremdsprache. Stuttgart: Ernst Klett Sprachen.

Menschen:

Evans, Sandra/Pude, Angela/Specht, Franz 2012: Menschen. Deutsch als Fremdsprache. Kursbuch A1. München: Hueber Verlag.

Glas-Peters, Sabine/Pude, Angela/Reimann, Monika 2012: Menschen. Deutsch als Fremdsprache. Arbeitsbuch A1. München: Hueber Verlag.

Habersack, Charlotte/Pude, Angela/Specht, Franz 2013: Menschen. Deutsch als Fremdsprache. Kursbuch A2. München: Hueber Verlag.

Breitsameter, Anna/Glas-Peters, Sabine/Pude, Angela 2013: Menschen. Deutsch als Fremdsprache. Arbeitsbuch A2. München: Hueber Verlag.

Braun-Podeschwa, Julia/Habersack, Charlotte/Pude, Angela 2014: Menschen. Deutsch als Fremdsprache. Kursbuch B1. München: Hueber Verlag.

Braun-Podeschwa, Julia/Habersack, Charlotte/Pude, Angela 2014: Menschen. Deutsch als Fremdsprache. Arbeitsbuch B1. München: Hueber Verlag.

Studio d:

Funk, Hermann/Kuhn, Christina/Demme, Silke 2005: studio d. Deutsch als Fremdsprache. Kurs- und Übungsbuch A1. Berlin: Cornelsen.

Funk, Hermann/Kuhn, Christina/Demme, Silke u. a. 2006: studio d. Deutsch als Fremdsprache. Kurs- und Übungsbuch A2. Berlin: Cornelsen.

Funk, Hermann/Kuhn, Christina/Demme, Silke/Winzer, Britta u. a. 2007: studio d. Deutsch als Fremdsprache. Kurs- und Übungsbuch B1. Berlin: Cornelsen.

استخدام أنواع النصوص فى التطبيق اللغوى كمدخل إلى نقل اللغة والثقافة

$$
\text { د. ديناته فريودنبرج فيندأيزن }
$$


مستخلص

تعلب معرفة أنواع النصوص عند تلقى و إنتاج النصوص لمتعلمى اللغة الأجنبية دورا

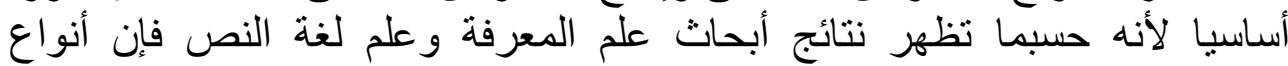

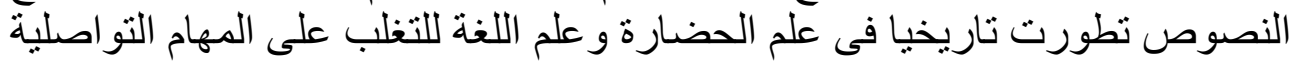

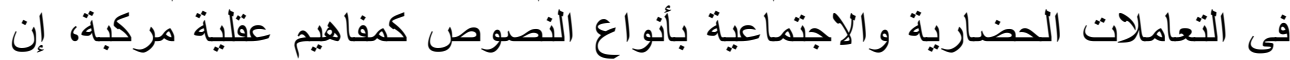

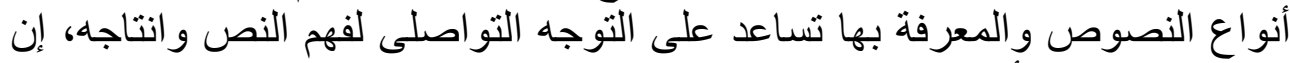

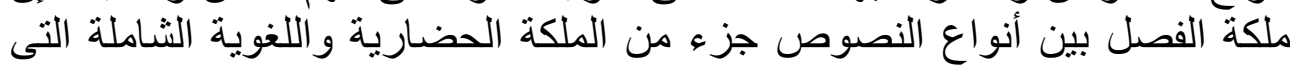

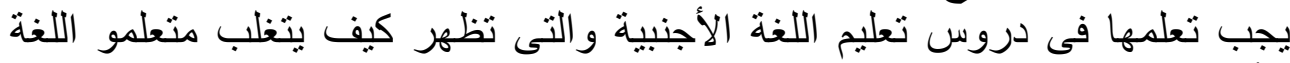

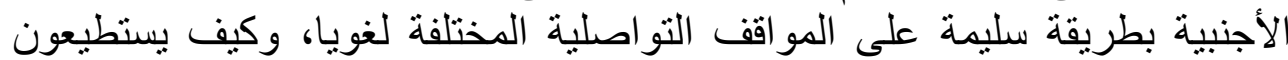

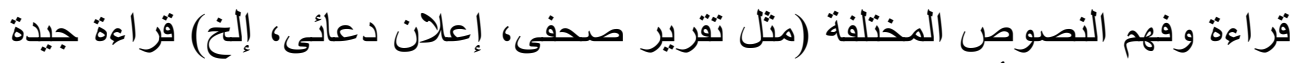
وفهم جيد، وإلى أى مدى ينجحون فى كتابة نصوص تناسب المتلقى والموقف

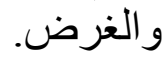

إلا أنه فى دروس اللغة الألمانية كلغة أجنبية وكتبها المتخصصة يبدو أنه لايزال

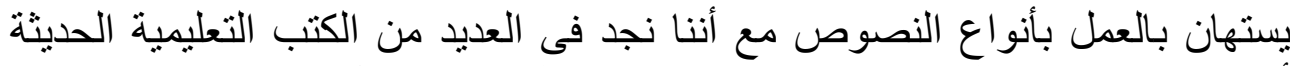

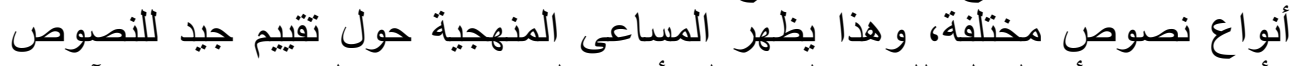
و أنواعها، إلا أن العمل اللغوى القائم على أنواع النصوص النص لايز ال ناقصـا حتى الآن.

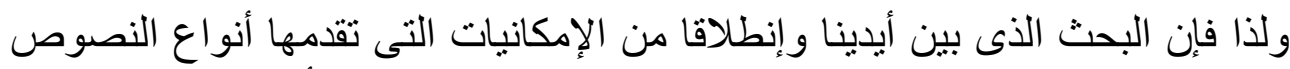

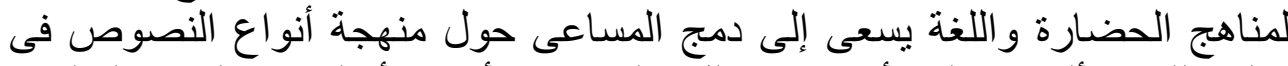

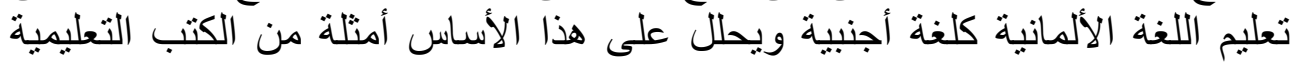
الحديثة تحليلا نقديا. 